\title{
On the correlation of elemental abundances with kinematics among galactic disk stars $\star, \star \star$
}

\author{
T. V. Mishenina ${ }^{1}$, C. Soubiran ${ }^{2}$, V. V. Kovtyukh ${ }^{1}$, and S. A. Korotin ${ }^{1}$ \\ 1 Astronomical Observatory of Odessa National University and Isaac Newton Institute of Chile, Odessa Branch, Ukraine \\ 2 Observatoire Aquitain des Sciences de l'Univers, CNRS UMR 5804, BP 89, 33270 Floirac, France
}

Received 6 October 2003 / Accepted 27 January 2004

\begin{abstract}
We have performed the detailed analysis of 174 high-resolution spectra of FGK dwarfs obtained with the ELODIE echelle spectrograph at the Observatoire de Haute-Provence. Abundances of $\mathrm{Fe}$, Si and $\mathrm{Ni}$ have been determined from equivalent widths under LTE approximation, whereas abundances of $\mathrm{Mg}$ have been determined under NLTE approximation using equivalent widths of 4 lines and profiles of 5 lines. Spatial velocities with an accuracy better than $1 \mathrm{~km} \mathrm{~s}^{-1}$, as well as orbits, have been computed for all stars. They have been used to define 2 subsamples kinematically representative of the thin disk and the thick disk in order to highlight their respective properties. A transition occurs at $[\mathrm{Fe} / \mathrm{H}]=-0.3$. Stars more metal-rich than this value have a flat distribution with $Z_{\max }<1 \mathrm{kpc}$ and $\sigma_{W}<20 \mathrm{~km} \mathrm{~s}^{-1}$, and a narrow distribution of $[\alpha / \mathrm{Fe}]$. There exist stars in this metallicity regime which cannot belong to the thin disk because of their excentric orbits, neither to the thick disk because of their low scale height. Several thin disk stars are identified down to $[\mathrm{Fe} / \mathrm{H}]=-0.80$. Their $\mathrm{Mg}$ enrichment is lower than thick disk stars with the same metallicity. We confirm from a larger sample the results of Feltzing et al. (2003) and Bensby et al. (2003) showing a decrease of $[\alpha / \mathrm{Fe}]$ with $[\mathrm{Fe} / \mathrm{H}]$ in the thick disk interpreted as the signature of the SNIa which have progressively enriched the ISM with iron. However our data suggest that the star formation in the thick disk stopped when the enrichment was $[\mathrm{Fe} / \mathrm{H}]=-0.30,[\mathrm{Mg} / \mathrm{Fe}]=+0.20,[\mathrm{Si} / \mathrm{Fe}]=+0.17$. A vertical gradient in $[\alpha / \mathrm{Fe}]$ may exist in the thick disk but should be confirmed with a larger sample. Finally we have identified 2 new candidates of the HR1614 moving group.
\end{abstract}

Key words. stars: fundamental parameters - stars: abundances - stars: kinematics - stars: atmospheres - Galaxy: stellar content

\section{Introduction}

The behaviour of stellar elemental abundances with age and kinematics, in various substructures of the Galaxy, puts important constraints to the construction of models of chemical and dynamical evolution of our galactic system. In this paper we put particular attention to the transition between the thin disk and the thick disk and to the abundances of the $\alpha$-elements $\mathrm{Mg}$ and $\mathrm{Si}$ and the iron-peak element $\mathrm{Ni}$. According to the current nucleosynthetic theory, $\alpha$-elements are being produced as a result of $\alpha$-capture reaction, taking place in the core of massive stars during their explosion as SN II (Burbidge et al. 1957). Fe is produced by both massive SN II and less massive SN Ia. It is expected that $2 / 3$ of the solar abundance of iron is produced by explosions of white dwarfs in double systems ( $\mathrm{SN} \mathrm{Ia),} \mathrm{1/3} \mathrm{by}$

Send offprint requests to: T. V. Mishenina,

e-mail: tamar@deneb.odessa.ua

* Based on spectra collected with the ELODIE spectrograph at the 1.93-m telescope of the Observatoire de Haute Provence (France).

$\star \star$ Tables 3 and 8 are only available in electronic form at the CDS via anonymous ftp to

cdsarc.u-strasbg.fr (130.79.128.5) or via

http://cdsweb.u-strasbg.fr/cgi-bin/qcat?J/A+A/418/551
SN II (Timmes et al. 1995). If the percentage of massive stars in the earlier Galaxy was higher than today, one can predict that the $\alpha / \mathrm{Fe}$ ratio is going to change over time. A well established observational fact shows that in old metal-poor stars of the Galaxy, $[\alpha / \mathrm{Fe}]$, in particular $[\mathrm{Mg} / \mathrm{Fe}]$, is overabundant relative to Sun's value (Wallerstein 1961; Gratton \& Sneden 1987; Magain 1989; Nissen et al. 1994; Fuhrmann et al. 1995 etc.). If the $\alpha$-element overabundance is a typical chemical pattern in halo stars in comparison with disk stars, there is a question whether there is a distinction in the $\mathrm{Mg}$ behaviour in other subsystems of the Galaxy, in particular in the thin and thick disks. Such a difference would have important consequences on the choice of the most probable scenario of formation of the thick disk (collapse, accretion etc.) and its timescale. Moreover $[\alpha / \mathrm{Fe}]$ could serve as an additional criterion for the deconvolution of thick and thin disk populations. It is especially important for the stars with metallicity in the interval of overlapping $[\mathrm{Fe} / \mathrm{H}]$ values $(-0.7<[\mathrm{Fe} / \mathrm{H}]<-0.3)$.

Several groups have recently attacked this problem by attempting to make consistent analyses of abundances of $\mathrm{Mg}$ and other elements in large $(>50)$ samples of stars of the thin and thick disks, using kinematics to distinguish the stellar populations. However, their results are ambiguous. Fuhrmann (1998) 
found the thick disk to be significantly older than the thin disk and its data exhibit an appreciable constant overabundance of $\mathrm{MgI}$ in the thick disk relative to the thin disk. He interprets the observed discontinuity in the chemical and kinematical behaviour of the thin disk and thick disk populations as a hiatus in star formation before the earliest stages of the thin disk. Mashonkina's group (Mashonkina \& Gehren 2000, 2001; Mashonkina et al. 2003) is in agreement with Fuhrmann concerning the discontinuity between the thin disk and the thick disk. They observe a step-like decrease of Eu/Ba ratio at the thick disk to thin disk transition and estimate that the thick disk formed from well mixed gas during a short timescale of $\sim 0.5$ Gyr. Gratton et al. (2000) argue in favour of a constant and significant overabundance of $\mathrm{Mg}$ with respect to $\mathrm{Fe}$ in the thick disk, a fast formation of the thick disk, a sudden decrease in star formation during the transition between the thick and thin disk phases and a formation scenario mixing dissipational collapse and accretion on similar time scales. Contrary to these studies, Chen et al. (2000) do not observe in their data any discontinuity between the thin disk and the thick disk. Their thick disk stars exhibit a slightly higher $\alpha /$ Fe ratio than the thin disk, but not exceeding +0.4 . Very recently Bensby et al. (2003) and Feltzing et al. (2003) published a convincing study showing that the thick disk extents at solar metallicities with an abundance trend clearly separated from the thin disk despite a large overlap in metallicity. A knee and decrease in $[\mathrm{Mg} / \mathrm{Fe}]$ vs. $[\mathrm{Fe} / \mathrm{H}]$ is interpreted as the signature of contribution from SNIa to the enrichment of the interstellar gas out of which the thick disk stars formed.

However these studies cannot be easily compared because they do not rely on the same definition of the thick disk. The thin disk and the thick disk are known to greatly overlap in kinematics and metallicity, and the selection of a sample representative of each population is not obvious. The use of a single criterion $\left(V_{\text {rot }}, Z_{\max }\right.$ or eccentricity of the orbit, $\left.[\mathrm{Fe} / \mathrm{H}], \ldots\right)$ is usually not sufficient for this task. In the last decade, studies of the thick disk kinematics have been numerous and its velocity distribution is now well constrained. This allows to make a rigourous classification of the stellar populations by computing the probability of each star of a sample to belong to the thin disk and the thick disk according to its velocity $(U, V, W)$, having first evaluated the selection biases which affect local samples. It is the way we have adopted in this paper to investigate the behaviour of $\mathrm{Mg}$ and $\mathrm{Si}$ (as alpha-element) and $\mathrm{Ni}$ (as iron-peak element) in the thin and thick disks.

In the past years, Mg abundances in stars have been determined in a wide range of metallicities and temperatures, either in LTE approximation or through detailed NLTE calculations. In the visible range, there are about $10 \mathrm{MgI}$ lines, but most of them are strong lines with equivalent width $(E W)$ greater than $200 \mathrm{~m} \AA$, and for such lines NLTE effects can be significant. As shown by several authors (Zhao \& Gehren 2000; Idiart \& Thévenin 2000; Shymanskaya et al. 2000), departures from LTE are observed in MgI lines in stars of various spectral types. We have therefore used the NLTE approach to determine $\mathrm{Mg}$ abundances more exactly. The lines that we have used for the other elements have small or moderate $E W$ and considering our temperature and metallicity interval, we have negleted NLTE corrections. As a matter of fact, in the Sun the abundance deviations due to NLTE effects are generally small and do not exceed 0.1 dex, as established in former calculations: 0.07 dex according to Shchukina \& Bueno (2001), <0.10 dex according to Gehren et al. (2001a,b) for FeI lines, and -0.01 dex for SiI lines (Wedemeyer 2001). Gratton et al. (1999) and Thévenin \& Idiart (1999) have investigated NLTE effects for iron lines in dwarfs and giants of different metallicity. In both papers, the dominating NLTE effect for Fe is the overionization by ultraviolet radiation (UV photons). Gratton et al. (1999) found that NLTE corrections may be neglected in most cases, including the stars on the main-sequence and red giant branch. Thévenin \& Idiart (1999) derived metallicity corrections of about 0.3 dex for stars with $[\mathrm{Fe} / \mathrm{H}]<-3.0$, however for stars with $[\mathrm{Fe} / \mathrm{H}]>-1$ this value does not exceed 0.1 dex. Abundances of $\mathrm{Fe}, \mathrm{Si}$ and $\mathrm{Ni}$ were therefore determined in LTE approximation.

This paper presents the determination and analysis of kinematical parameters and abundances of $\mathrm{Mg}, \mathrm{Si}$ and $\mathrm{Ni}$ for 174 dwarf stars in the domain of $-1.0<[\mathrm{Fe} / \mathrm{H}]<+0.3$. This study was carried out using a homogeneous spectral material, uniform methods of treatment and NLTE calculations for $9 \mathrm{MgI}$ lines. Two subsamples have been defined on the basis of kinematics to be representative of the thin disk and the thick disk and are used to highlight the chemical behaviour of the two stellar populations.

\section{Observations and reduction}

All the spectra used in this paper are extracted from the most recent version of the library of stellar spectra collected with the ELODIE echelle spectrograph at the Observatoire de HauteProvence by Soubiran et al. (1998) and Prugniel \& Soubiran (2001). The performances of the instrument mounted on the $193 \mathrm{~cm}$ telescope, are described in Baranne et al. (1996). ELODIE is a very stable instrument, build to monitor radial velocity oscillations of stars with exoplanets, at a resolving power of 42000 in the wavelength range $\lambda \lambda 3850-6800 \AA$. Spectrum extraction, wavelength calibration and radial velocity measurement have been performed at the telescope with the on-line data reduction software while straightening of the orders, removing of cosmic ray hits, bad pixels and telluric lines were performed as described in Katz et al. (1998).

All the spectra of the library have been parametrized in terms of $\left(T_{\mathrm{eff}}, \log g,[\mathrm{Fe} / \mathrm{H}]\right)$, either from the literature or internally with the TGMET code (Katz et al. 1998). This allowed us to select a set of 174 FGK dwarfs and subgiants $(3.7<\log g<4.6)$ for this study, with metallicities in the range $-1<[\mathrm{Fe} / \mathrm{H}]<+0.3$. Several solar spectra taken on the Moon and asteroids were also considered as references. The selected spectra have a signal to noise ratio $(S / N)$ at $5500 \AA$ ranging from 100 to 300 .

The continuum level drawing and equivalent width measurements were carried out by us using DECH20 code (Galazutdinov 1992). Equivalent widths of lines were measured by Gaussian function fitting. Their accuracy was estimated by comparing our measurements on solar spectra to those obtained by other authors. The mean difference with 


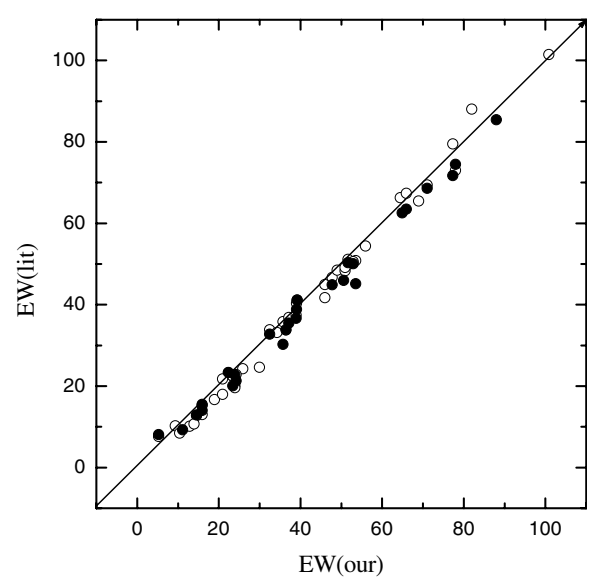

Fig. 1. Comparison of solar $E W$ measured in this study with those from the literature (Edvardsson et al. 1993 - filled circles, Reddy et al. 2002 - open circles).

Edvardsson et al. (1993) is: $E W_{\mathrm{Edv}}-E W_{\text {our }}=-2.3(\sigma=$ 2.3) $\mathrm{m} \AA$ for 27 lines of FeI, FeII, SiI and NiI in common; and with Reddy et al. (2002): $E W_{\text {Red }}-E W_{\text {our }}=-1.2(\sigma=2.1) \mathrm{m} \AA$ for 47 lines in common. The comparison is shown in Fig. 1.

\section{Atmospheric parameters}

In order to perform reliable abundance determinations, it is crucial to derive accurate stellar parameters, especially effective temperatures $T_{\text {eff }} . V-K$ or IR flux methods are often advocated as the best (Asplund 2003) but require homogeneous infrared observations which are not available for all stars. Recently our group has improved the line-depth ratio technique pioneered by Gray (1994), leading to high precision $T_{\text {eff }}$ for most of our program stars (Kovtyukh et al. 2003). This method, relying on ratios of the measured central depths of lines having very different functional dependences on $T_{\text {eff }}$, is independent of interstellar reddening and takes into account the individual characteristics of the star's atmosphere. Briefly, a set of 105 relations was obtained, the mean random error of a single calibration being $60-70 \mathrm{~K}(40-45 \mathrm{~K}$ in the most and $90-95 \mathrm{~K}$ in the least accurate cases). The use of $\sim 70-100$ calibrations per spectrum reduced the uncertainty to 5-7 K. These 105 relations were obtained from 92 lines, 45 with low $(\chi<2.77 \mathrm{eV})$ and 47 with high $(\chi>4.08 \mathrm{eV})$ excitation potentials, calibrated from 45 reference stars in common with Alonso et al. (1996), Blackwell \& Lynas-Gray (1998) and di Benedetto (1998). The zero-point of the temperature scale was directly adjusted to the Sun, based on 11 solar reflection spectra taken with ELODIE, leading to the uncertainty in the zero-point of about $1 \mathrm{~K}$. Judging by the small scatter in our final calibrations and $T_{\text {eff }}$, the selected combinations are only weakly sensitive to effects like rotation, micro- and macroturbulence, non-LTE and other. The application range of the line-depth method is $-0.5<[\mathrm{Fe} / \mathrm{H}]<+0.5$.

For the most metal-poor stars, $T_{\text {eff }}$ was determined earlier (Mishenina \& Kovthyukh 2001). The $\mathrm{H}_{\alpha}$ line-wing fitting was used for stars studed in this work. None of the stars from Mishenina \& Kovthyukh (2001) had their temperature measured by line depth ratio because they are too metal poor. In order to testify that the temperature scales adopted in

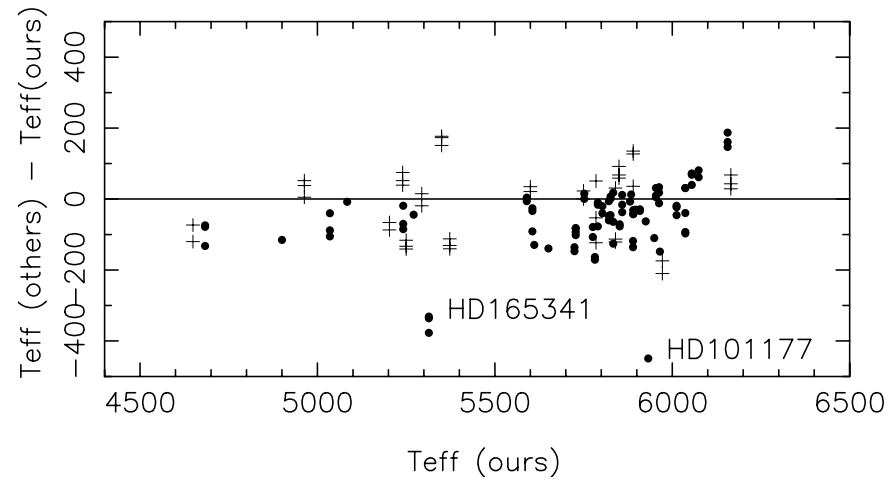

Fig. 2. Comparison of our adopted temperatures with those estimated by Alonso et al. (1996), Blackwell \& Lynas-Gray (1998) and di Benedetto (1998). Crosses indicate stars with $[\mathrm{Fe} / \mathrm{H}]<-0.5$. The two outliers HD 101177 and HD 165341 are discussed in the text. HD 165341 was measured by Alonso et al., that is why there are 3 points for the same star.

Table 1. Mean difference and standard deviation between our temperatures and those estimated by Alonso et al. (1996), Blackwell \& Lynas-Gray (1998) and di Benedetto (1998) for 40 stars in common.

\begin{tabular}{lccc}
\hline \hline Reference & $N$ & $\Delta T_{\text {eff }}(\mathrm{K})$ & $\sigma(\mathrm{K})$ \\
\hline Alonso et al. (1996) & 43 & 31 & 84 \\
Blackwell \& Lynas-Gray (1998) & 41 & 29 & 84 \\
di Benedetto (1998) & 41 & 23 & 78 \\
\hline
\end{tabular}

Mishenina \& Kovthyukh (2001) and Kovtyukh et al. (2003) are consistent, we show in Fig. 2 our adopted temperatures versus those estimated by Alonso et al. (1996), Blackwell \& Lynas-Gray (1998) and di Benedetto (1998). Our temperature scale is slightly hotter than their by $\sim 20-30 \mathrm{~K}$, as mentioned in Kovtyukh et al. (2003), but the dispersion is satisfactory ( $\sim 80 \mathrm{~K}$, Table 1$)$. We have checked the 2 outliers HD 101177 and HD 165341. We are certain that Alonso et al.'s temperature for HD $101177(5483 \mathrm{~K})$ is in error because recently Heiter \& Luck (2003) determined a temperature of $6000 \mathrm{~K}$ similar to ours $(5932 \mathrm{~K})$, a value confirmed by the $\mathrm{H}_{\alpha}$ profile. HD 165341 is a variable, active, rotating spectroscopic binary rending its photometric measurements suspicious. On the basis of IR photometry, Alonso et al. (1996), Blackwell \& Lynas-Gray (1998) and di Benedetto (1998) determined respectively 4978 K, 4983 K, 4937 K. Our determination of $5314 \mathrm{~K}$ agrees well with that of Zboril \& Byrne (1998) who find $T_{\text {eff }}=5260 \mathrm{~K}$.

Finally, Fig. 3 shows a general lack of correlation between the $\mathrm{Fe}$ and $\mathrm{Mg}$ abundances and $T_{\text {eff }}$ that testifies to a correct choice of the effective temperatures.

The two most commonly used techniques to determine surface gravities are the ionization balance of neutral and ionized species and the fundamental relation which expresses the gravity as a function of mass, temperature and bolometric absolute magnitude deduced from parallaxe. For the latter method, stellar masses can be estimated from evolutionary tracks, but metallicities and eventual $\alpha$-enhancement have to be known first. We therefore choosed to derive $\log g$ from ionization balance, a method which might be affected by NLTE effects. 

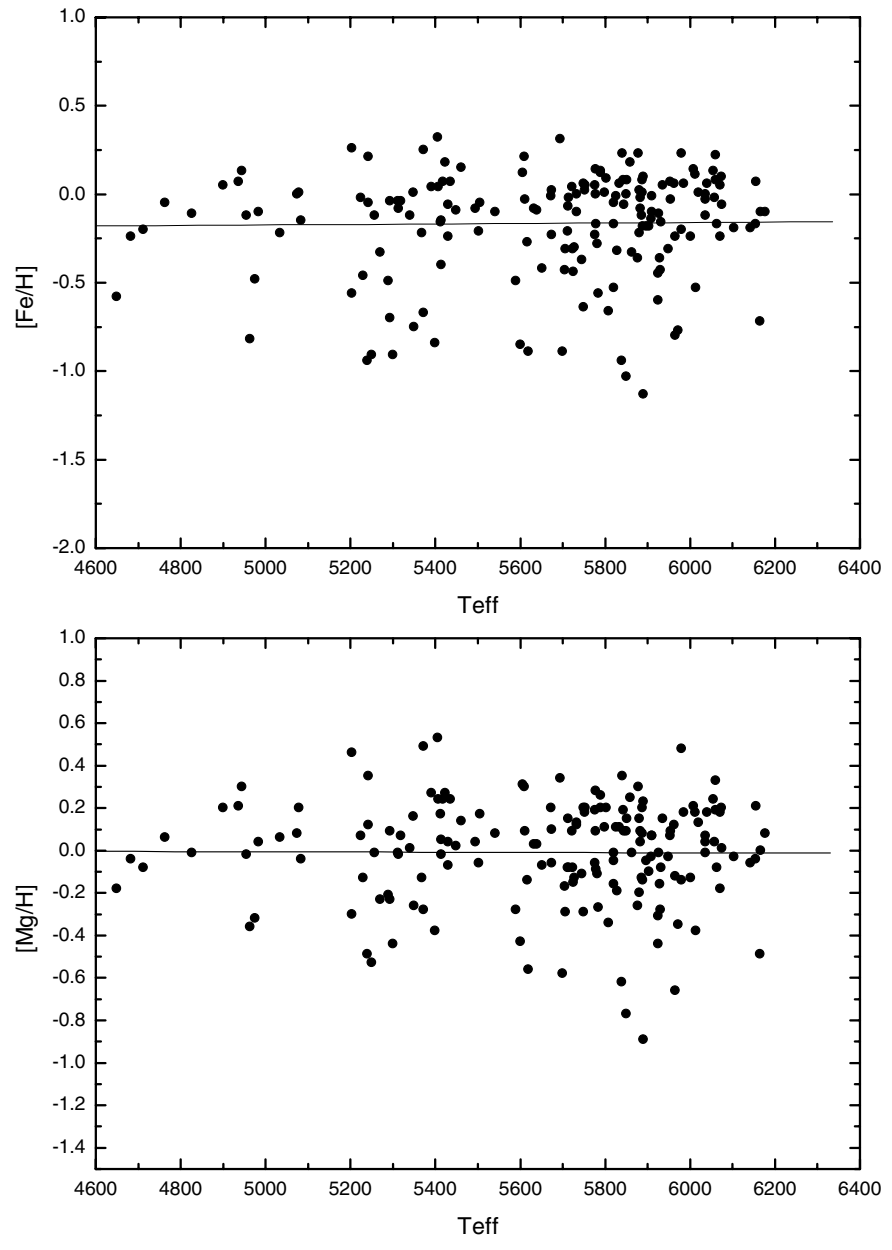

Fig. 3. Abundances of $\mathrm{Fe}$ and $\mathrm{Mg}$ vs. $T_{\text {eff }}$.

However, a detailed study of surface gravities derived by different procedures was preformed by Allende Prieto et al. (1999) who concluded that astrometric and spectroscopic (iron ionization balance) gravities were in good agreement in the metallicity range $-1.0<[\mathrm{Fe} / \mathrm{H}]<+0.3$. We compared our adopted surface gravities to those determined astrometrically by Allende Prieto et al. (1999) and obtain a mean difference and standard deviation of -0.01 and 0.15 respectively for 39 stars in common. This is consistent with an accuracy of 0.1 dex on our estimated spectroscopic gravities. As an additional criterion of the reliable choice of $\log g$ we used the wings-fitting for the MgIb lines. For all stars the difference does not exceed 0.2 dex (such difference was detected only for a few program stars).

Microturbulent velocities $V_{\mathrm{t}}$ were determined by forcing the abundances determined from individual $\mathrm{FeI}$ lines to be independent of equivalent width. Starting with $V_{\mathrm{t}}=1.1 \mathrm{~km} \mathrm{~s}^{-1}$, we varied it until abundances computed for FeI lines $(20 \mathrm{~m} \AA<$ $E W<150 \mathrm{~m} \AA$ ) and plotted as a function of $E W$-s showed a zero slope. The precision of the microturbulent velocity determination is $0.2 \mathrm{~km} \mathrm{~s}^{-1}$.

The abundance of iron relative to solar one $[\mathrm{Fe} / \mathrm{H}]=$ $\log (\mathrm{Fe} / \mathrm{H})_{*}-\log (\mathrm{Fe} / \mathrm{H})_{\odot}$ was used as the metallicity parameter of a star and was obtained from FeI lines.
Table 2. Number of stars in common, mean difference and standard deviation of parameter comparison with 5 other studies.

\begin{tabular}{rrrrrrcc}
\hline \hline$N$ & $\Delta T_{\text {eff }}$ & $\sigma$ & $\Delta \log g$ & $\sigma$ & $\Delta[\mathrm{Fe} / \mathrm{H}]$ & $\sigma$ & Ref $^{*}$ \\
\hline 37 & -2 & 78 & 0.03 & 0.17 & -0.07 & 0.08 & 1 \\
39 & -28 & 82 & 0.04 & 0.22 & -0.04 & 0.10 & 2 \\
18 & 10 & 75 & 0.04 & 0.15 & -0.01 & 0.09 & 3 \\
17 & -99 & 56 & -0.06 & 0.13 & -0.08 & 0.06 & 4 \\
12 & -15 & 74 & 0.05 & 0.16 & 0.04 & 0.09 & 5 \\
\hline
\end{tabular}

* 1 - Edvardsson et al. (1993); 2 - Gratton et al. (1996); 3 - Fuhrmann (1998); 4 - Chen et al. (2000); 5 - Fulbright (2000).

The adopted parameters of the target stars are given in Table 8 . To check the adopted model atmosphere parameters, we compared our values with those derived by Edvardsson et al. (1993), Gratton et al. (1996), Fuhrmann (1998), Chen et al. (2000) and Fulbright (2000). The mean differences (other - us) and standard deviations are given in Table 2. On the whole, the agreement is rougly good.

\section{Abundance determination}

\subsection{Fe, Si, Ni abundances}

$\mathrm{Fe}, \mathrm{Si}, \mathrm{Ni}$ abundances were determined from an LTE analysis of equivalent widths using the WIDTH9 code and the atmosphere models by Kurucz (1993). Appropriate models for each star were derived by means of standard interpolation through $T_{\text {eff }}$ and $\log g$. The model metallicities were taken with an accuracy of \pm 0.25 dex around the TGMET first guess. Abundances of the investigated elements $\mathrm{Fe}, \mathrm{Si}, \mathrm{Ni}$ were carried out from a differential analysis relative to the Sun. Solar abundances of $\mathrm{Fe}, \mathrm{Si}$ and $\mathrm{Ni}$ were calculated with our solar $E W \mathrm{~s}$ and the oscillator strengths $\log g f$ from Kovtyukh \& Andrievsky (1999), who derived $\log g f$ for 565 lines of the 27 chemical elements using solar spectra. We determined: $\log A(\mathrm{Fe})=7.55$, $\log A(\mathrm{Si})=7.55, \log A(\mathrm{Ni})=6.25$, where $\log (\mathrm{H})=12(\mathrm{ac}-$ cording to Grevesse \& Sauval 1998: $\log A(\mathrm{Fe})=7.50$, $\log A(\mathrm{Si})=7.55, \log A(\mathrm{Mg})=7.58, \log A(\mathrm{Ni})=6.25)$.

Depending on authors, the solar iron abundance varies from 7.44 to 7.64 (Asplund et al. 2000; Blackwell et al. 1995; Gehren et al. 2000a; Grevesse \& Sauval 1998; Shchukina \& Bueno 2001, etc.). This disagreement is caused by several factors: the systems of oscillators strengths, the models of solar atmosphere (empirical, hydrodynamical, LTE or NLTE assumptions), the solar spectrum LTE or NLTE synthesis etc. Using laboratory $\log g f$, Blackwell et al. (1995) have obtained a solar iron abundance of 7.64. From calculated and laboratory $\log g f$, Gehren (2001a) found an abundance 7.50 and 7.56 from Fe II lines, and 7.47 and 7.56 from Fe I lines. The detailed hydrodynamical models of the Sun result in the solar value of iron abundance of 7.44 (Fe I lines) and 7.45 (Fe II lines) (Asplund et al. 2000). In the same time of the meteoretic value of the iron abundance is 7.50 (Grevesse \& Sauval 1998). The same value was found for the solar atmosphere by Shchukina \& Bueno (2001) using the NLTE approximation and hydrodynamical solar model. The solar silicon abundance obtained 
Table 3. List of $\mathrm{Fe}, \mathrm{Si}$ and Ni lines, atomic parameters and solar $E W$. Only available at the CDS.

within the framework of the hydrodynamical models (Asplund $2000)$ is sligtly lower (7.51) than the meteoric silicon abundance (7.55) (Grevesse \& Sauval 1998). To reduce the influence of the used systems of oscillators strengths, atmosphere models and instrumental errors we used a differential method of abundance determination.

The list of $\mathrm{Fe}, \mathrm{Si}$ and $\mathrm{Ni}$ lines, atomic parameters and solar $E W$ are given in Table 3. Relative abundances of $\mathrm{Fe}, \mathrm{Si}$ and $\mathrm{Ni}$ are given in Table 8.

\subsection{NLTE calculations for $M g$}

The determination of $\mathrm{Mg}$ abundance was carried out through detailed NLTE calculations using equivalent widths of 4 lines $(\lambda \lambda 4730,5711,6318,6319 \AA)$ and profiles of 5 lines $(\lambda \lambda 4571$, 4703, 5172, 5183, $5528 \AA$ ).

NLTE abundances of Mg were determined with the help of a modified version of the MULTI code of Carlsson (1986) described in Korotin et al. (1999a,b). We have included in the code opacity sources from the ATLAS9 program (Kurucz 1992). This enables a much more accurate calculation of the continuum opacity and intensity distribution in the UV region which is extremely important in the correct determination of the radiative rates of $b-f$ transitions. For $b-b$ transitions include only continuum opacity and for $b-f$ transitions include continuum both opacities and line opacities from ATLAS9. A simultaneous solution of the radiative transfer and statistical equilibrium equations has been performed in the approximation of complete frequency redistribution for all lines.

We employed the model of magnesium atom consisting of 97 levels: 84 levels of $\mathrm{Mg}$ I, 12 levels of $\mathrm{Mg}$ II and a ground state of Mg III. They were selected from works of Martin \& Zalubas (1980) and Biemont \& Brault (1986). A detailed structure of the multiplets was ignored and each $L S$ multiplet was considered as a single term. The fine structure was taken into account only for a level $3 \mathrm{~s} 3 \mathrm{p}^{3} \mathrm{P}^{0}$, since it is closely connected to the most important transitions in magnesium atom ( $\lambda \lambda 2778-2782 \AA, 3829-3838 \AA, 5167 \AA, 5172 \AA)$.

Within the described system of the magnesium atom levels, we considered the radiative transitions between the first 59 levels of Mg I and ground level of Mg II. Transitions between the rest levels were not taken into account and they were used only in the equations of particle number conservation.

Only transitions with $\lambda<100000 \AA$ were selected for the analysis. All $424 b-b$ transitions were included in the linearization procedure.

Grotrian diagram for the MgI are displayed in Fig. 4.

Photoionization cross-sections were mainly taken from the Opacity Project (Yan et al. 1987) keeping a detailed structure of their frequency dependence, including resonances. For some important $b-f$ transitions, the cross-section structure is extremely complicated making it difficult to describe it using only simple approximations like $\sim v^{-3}$.

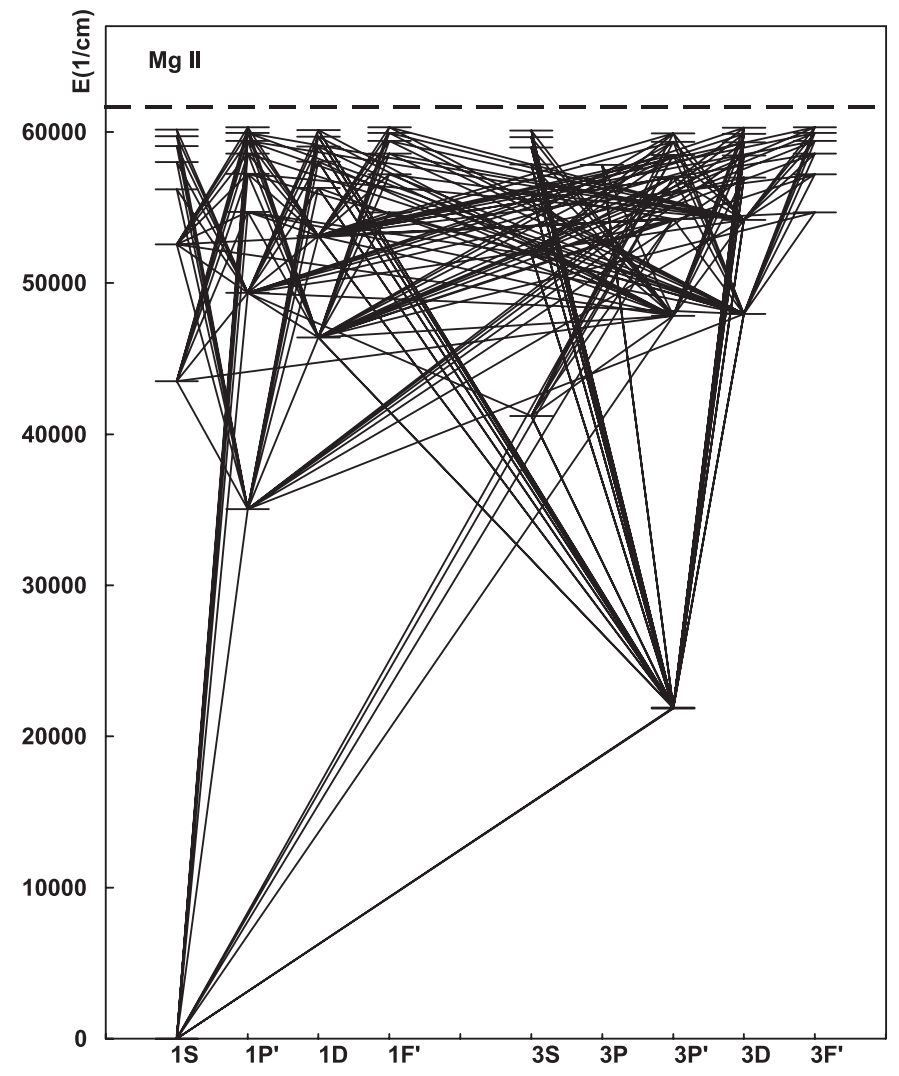

Fig. 4. Grotrian diagram for MgI. Displayed are the radiative transitions treated explicity in the non-LTE calculations.

Oscillator strengths were selected from the extensive compilative catalogue by Hirata \& Horaguchi (1994). Some information was obtained through the Opacity Project. As we ignored a multiple structure of all the levels, the oscillator strengths for each averaged transition were calculated as $f=\frac{\sum g_{i} f_{i}}{\sum g_{i}}$.

After the combined solution of radiative transfer and statistical equilibrium equations, the averaged levels have been split with respect to multiplet structure, then level populations were redistributed proportionally to the statistical weights of the corresponding sublevels and finally the lines of the interest were studied.

Electron impact ionization was described using Seaton's formula (Seaton 1962). Collision rate between ground level $\mathrm{Mg}$ I and ground level of $\mathrm{Mg}$ II were approximated by use of the fits from Voronov (1997). For electron impact excitation for all allowed $b-b$ transitions we used the van Regemorter (1962) formula. Collisional rates for the forbidden transitions were calculated by using the semiempirical formula (Allen 1973), with a collisional force of $=1$.

Inelastic collisions with hydrogen may play a significant role in the atmospheres of cool stars. We took into account this effect with the help of Drawin's formulas (1968) offered Steenbock \& Holweger (1984), with a correction factor of $1 / 3$. Nevertheless, as it was shown in our test calculation, the influence of such collisions is negligible for the stars considered in our paper. In particular, the variation of the correction 
Table 4. Atomic data for the $\mathrm{Mg}$ lines.

\begin{tabular}{ccccc}
\hline \hline Lambda & $\chi(\mathrm{eV})$ & $\log g f$ & $\Delta C_{6}$ & $\begin{array}{c}\Delta C_{6} \\
\text { (Barklem et al. 2000) }\end{array}$ \\
\hline 4571.10 & 0.00 & -5.79 & 1.72 & \\
4702.99 & 4.35 & -0.52 & 0.74 & 1.33 \\
4730.03 & 4.35 & -2.32 & 1.75 & \\
5172.68 & 2.71 & -0.38 & 0.74 & 0.85 \\
5183.60 & 2.71 & -0.16 & 0.72 & 0.85 \\
5528.40 & 4.35 & -0.49 & 0.44 & 0.95 \\
5711.09 & 4.35 & -1.72 & 0.60 & \\
6318.72 & 5.11 & -1.97 & 1.19 & \\
6319.24 & 5.11 & -2.18 & 1.19 & \\
6319.49 & 5.11 & -2.61 & 1.19 & \\
\hline
\end{tabular}

factor from 0 to 1 results in a change of the equivalent width less than $0.5 \%$.

For all the transitions we also took into account such broadening parameters of lines as radiative damping, Stark effect, van der Waals damping and microturbulent velocity. For temperatures, considered by us, the influence of Stark effect is small.

The main influence on the profile was exerted by van der Waals broadening. The Unsöld's (1955) formula which is widely used to allow for this effect, is known to yield somewhat understimated coefficients $C_{6}$. To refine these coefficients, we conducted a special comparison of the observed and calculated line profiles in solar spectrum using method Shimanskaya et al. (2000). The Solar Flux Atlas of Kurucz et al. (1984) and Kurucz's model of the solar atmosphere (1992) were used. To take into account the chromospheric growth of temperature, this model was completed by a model of the solar chromosphere from work of Maltby et al. (1986). Nevertheless, the influence of a chromosphere on the equivalent width of considered lines was insignificant (less than $1 \%$ ).

The corrections $\Delta \log C_{6}$ to the classic Unsöld (1955) collisional damping constant derived from solar line wing fitting are given in Table 4. These values are comparable for common lines with results obtained in the paper of Shimanskaya et al. (2000). Similar values were obtained by Barklem et al. (2000). For example, their correction factors for the lines $\lambda \lambda 5172,5183 \AA$ are $\Delta \log C_{6}=0.85$. Oscillator strengths for observed lines were selected from the compilative catalogue by Hirata \& Horaguchi (1994).

The estimates obtained from the NLTE profile analysis of the $\mathrm{MgI}$ lines in the solar spectrum give the solar magnesium content $(\mathrm{Mg} / \mathrm{H})=7.57 \pm 0.02$ which agrees well with the determination of Grevesse \& Sauval (1998) and Shimanskaya, Mashonkina \& Sakhibullin (2000), who derived $(\mathrm{Mg} / \mathrm{H})=7.58$.

The comparison the NLTE profiles with those observed are presented in Fig. 5. NLTE abundances of $\mathrm{Mg}$ are given in Table 8.

The NLTE effects for the used lines appeared to be very small (less than 0.05 dex). The similar results were obtained in Shimanskaya et al. (2000).
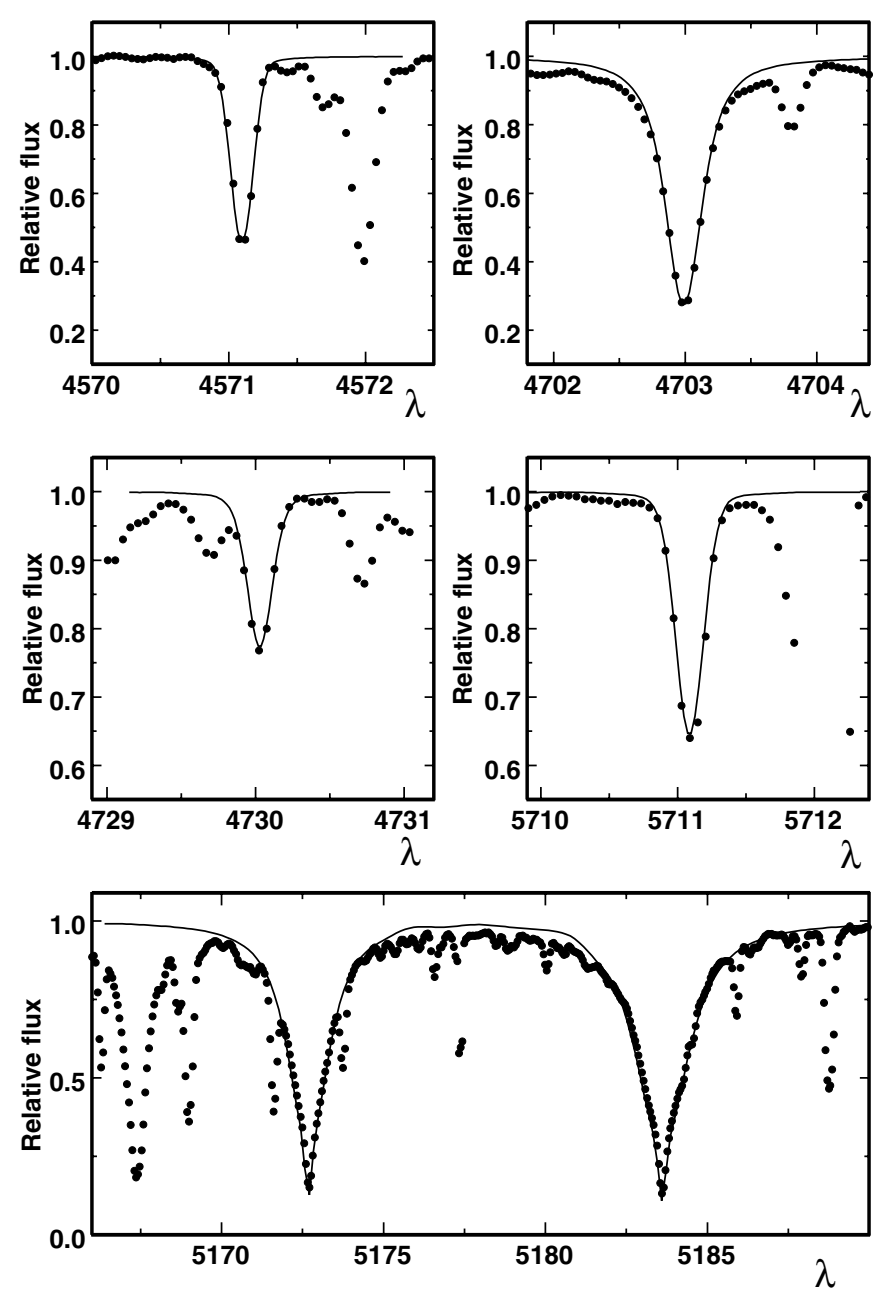

Fig. 5. Comparison of synthetic NLTE profiles (continuous line) of the MgI lines to the observed spectrum of HD 224930 (dots).

\section{Error analysis}

Average standard deviations of abundances obtained from 72-265 lines of Fe I (the number of used lines differs from star to star), 11-24 lines of Si I and 12-17 lines of Ni I are 0.10, 0.08 and 0.08 respectively. Final errors in abundances result mainly from errors in the choice of parameters of the model atmospheres and in equivalent width measurements (Gaussian fitting, placement of the continuum) in the case of $\mathrm{Fe}, \mathrm{Si}$, and $\mathrm{Ni}$ or fitting a synthetic spectrum in the case of $\mathrm{Mg}$. Table 5 lists the errors obtained when changing the atmospheric parameters by $\Delta T_{\text {eff }}=-100 \mathrm{~K} ; \Delta \log g=+0.2 ; \Delta V_{\mathrm{t}}=+0.2 \mathrm{~km} \mathrm{~s}^{-1}$; $\Delta[\mathrm{Fe} / \mathrm{H}]= \pm 0.25$ and by assuming an uncertainty of $\pm 2 \mathrm{~m} \AA$ in the $E W$. These values were adopted taking into account the intrinsic accuracy of the atmospheric parameter determination, the processing of the spectra and the comparison of our parameter definition with those of other authors. This test has been performed for 3 stars with different characteristics.

As seen in Table 5, the total uncertainty reaches 0.12 dex for iron abundance determined from II species and $0.10 \mathrm{dex}$ in the case of I species. The standard deviation obtained by comparing our $[\mathrm{Fe} / \mathrm{H}]$ determinations to those obtained by other authors (Table 2) shows that we are consistent with them at a level lower than 0.10 dex. 
Table 5. Effects of uncertainties of model parameters and $E W$ measurements on the derived abundances.

\begin{tabular}{|c|c|c|c|c|c|c|}
\hline & \multicolumn{2}{|c|}{$\overline{~ H D ~} 004614$} & \multicolumn{3}{|c|}{ 5965/4.4/1.1/-0.24 } & \multirow[b]{2}{*}{ Total } \\
\hline El & $\overline{T_{\text {eff }}}$ & $\log g$ & $\overline{V_{\mathrm{t}}}$ & $E W$ & {$[\mathrm{Fe} / \mathrm{H}]$} & \\
\hline $\mathrm{FeI}$ & 0.07 & 0.02 & 0.04 & 0.04 & 0.00 & 0.09 \\
\hline FeII & -0.02 & -0.08 & 0.02 & 0.04 & 0.03 & 0.10 \\
\hline MgI & 0.05 & 0.02 & 0.02 & 0.03 & 0.00 & 0.06 \\
\hline SiI & 0.02 & 0.01 & 0.02 & 0.03 & 0.01 & 0.05 \\
\hline \multirow[t]{2}{*}{$\mathrm{NiI}$} & 0.07 & 0.00 & 0.03 & 0.04 & 0.00 & 0.09 \\
\hline & \multicolumn{2}{|c|}{ HD 022879} & \multicolumn{3}{|c|}{$5972 / 4.5 / 1.1 /-0.77$} & \\
\hline FeI & 0.05 & 0.02 & 0.02 & 0.08 & 0.00 & 0.10 \\
\hline FeII & -0.01 & -0.09 & 0.01 & 0.07 & 0.02 & 0.12 \\
\hline MgI & 0.07 & 0.03 & 0.02 & 0.04 & 0.00 & 0.09 \\
\hline SiI & 0.02 & -0.01 & 0.00 & 0.05 & 0.00 & 0.05 \\
\hline \multirow[t]{2}{*}{ NiI } & 0.07 & 0.01 & 0.01 & 0.06 & 0.00 & 0.09 \\
\hline & \multicolumn{2}{|c|}{ HD 117176} & \multicolumn{3}{|c|}{$5611 / 4.0 / 1.0 /-0.03$} & \\
\hline FeI & 0.07 & 0.03 & 0.04 & 0.04 & 0.01 & 0.10 \\
\hline FeII & -0.04 & -0.10 & 0.02 & 0.04 & 0.04 & 0.12 \\
\hline MgI & 0.06 & 0.03 & 0.03 & 0.03 & 0.00 & 0.08 \\
\hline SiI & 0.01 & -0.01 & 0.02 & 0.03 & 0.02 & 0.05 \\
\hline NiI & 0.06 & 0.00 & 0.03 & 0.04 & 0.01 & 0.08 \\
\hline
\end{tabular}

(1) $-\Delta T_{\text {eff }}=-100 \mathrm{~K}$;

(2) $-\Delta \log g=+0.2$;

(3) $-\Delta V_{\mathrm{t}}=+0.2 \mathrm{~km} \mathrm{~s}^{-1}$;

(4) $-\Delta[\mathrm{Fe} / \mathrm{H}]=-0.25 \mathrm{dex}$;

(5) $-\Delta E W= \pm 2 \mathrm{~m} \AA$;

(6) - Total error.

Table 6. Abundance $[\mathrm{Mg} / \mathrm{H}],[\mathrm{Si} / \mathrm{Fe}],[\mathrm{Ni} / \mathrm{Fe}]$ comparison with other authors.

\begin{tabular}{cccccccc}
\hline \hline$N$ & $\Delta_{\mathrm{Mg}}$ & $\sigma$ & $\Delta_{\mathrm{Si}}$ & $\sigma$ & $\Delta_{\mathrm{Ni}}$ & $\sigma$ & Ref $^{*}$ \\
\hline 18 & 0.03 & 0.09 & & & & & 1 \\
10 & 0.04 & 0.09 & & & & & 2 \\
37 & & & -0.05 & 0.05 & 0.01 & 0.05 & 3 \\
17 & & & -0.04 & 0.05 & 0.02 & 0.03 & 4 \\
5 & & & 0.01 & 0.08 & -0.05 & 0.09 & 5 \\
\hline
\end{tabular}

* 1 - Idiard \& Thévenin (2000); 2 - Gratton et al. (2003); 3 - Edvardsson et al. (1993); 4 - Chen et al. (2000); 5 - Reddy et al. (2003).

For $\mathrm{Mg}, \mathrm{Si}$ and $\mathrm{Ni}$, the total uncertainty due to parameter and $E W$ errors is 0.08 dex, 0.05 dex and 0.09 dex respectively. We have also compared our $\mathrm{Mg}, \mathrm{Si}$ and $\mathrm{Ni}$ abundances with those determined by other authors. For $[\mathrm{Mg} / \mathrm{H}]$, we have carried out the comparison with Idiart \& Thévenin (2000) and Gratton et al. (2003) who have also used the NLTE approach. Our $[\mathrm{Si} / \mathrm{Fe}]$ and $[\mathrm{Ni} / \mathrm{Fe}]$ determinations have been compared to those of Edvardsson et al. (1993), Reddy et al. (2002) and Chen et al. (2000). The results of comparisons are listed in Table 6. The mean differences are lower than 0.05 and the standard deviations lower than $0.1 \mathrm{dex}$ for all elements proving the good consistency of our study with previous works relying on similar methods.
Table 7. Kinematical parameters adopted for the thin disk and the thick disk from Soubiran et al. (2003).

\begin{tabular}{ccc}
\hline \hline & Thin disk & Thick disk \\
\hline$V_{\text {lag }}\left(\mathrm{km} \mathrm{s}^{-1}\right)$ & -15 & -51 \\
$\sigma_{U}\left(\mathrm{~km} \mathrm{~s}^{-1}\right)$ & 39 & 63 \\
$\sigma_{V}\left(\mathrm{~km} \mathrm{~s}^{-1}\right)$ & 20 & 39 \\
$\sigma_{W}\left(\mathrm{~km} \mathrm{~s}^{-1}\right)$ & 20 & 39 \\
\hline
\end{tabular}

\section{Stellar kinematics, metallicity, elemental abundances}

All the selected stars are bright and nearby enough to have parallaxes and proper motions measured by Hipparcos (ESA 1997). These astrometric quantities have been combined with radial velocties measured on the ELODIE spectra by crosscorrelation (with an accuracy better than $100 \mathrm{~m} \mathrm{~s}^{-1}$ ) to compute the 3 components $(U, V, W)$ of the spatial velocities with respect to the Sun. Combining the measurement errors on parallaxes, proper motions and radial velocities, the resulting errors on velocities are of the order of $1 \mathrm{~km} \mathrm{~s}^{-1}$.

Our first concern was to analyse the content of our sample in terms of stellar populations. As we were interested in the characterization of abundance patterns in the thin disk and the thick disk, we have selected our sample to span the metallicity range $-1.0<[\mathrm{Fe} / \mathrm{H}]<+0.3$ in order to define 2 subsamples representative of these two populations. A discrimination of thin disk and thick disk stars is possible using the fact that the two disks are known to be distinct by their spatial distribution and local density, and by their velocity, metallicity and age distributions. However we have performed this classification with a pure kinematical appoach because velocity distributions are the best constrained from observations reported in the literature. If the thin disk velocity distribution is well known thanks to Hipparcos data, authors do not yet agree on the properties of the thick disk. A review of the current knowledge of the thick disk is given in Norris (1999). There is still a controversy between the adherents of a flat and dense thick disk, with typical scale height of $800 \mathrm{pc}$ and local relative density of 6-7\% (Reylé \& Robin 2001) to $15 \%$ (Soubiran et al. 2003), and the adherents of a thick disk with a higher scale height, typically $1300 \mathrm{pc}$ and a lower local relative density, of the order of $2 \%$ (Reid \& Majewski 1993). Velocity dispersions are generally found to span typical values between 30 and $50 \mathrm{~km} \mathrm{~s}^{-1}$, with an asymmetric drift between -20 and $-80 \mathrm{~km} \mathrm{~s}^{-1}$. Recently, Soubiran et al. (2003) determined the kinematical parameters of the thin disk and the thick disk from an unbiased sample of clump giants. These values, listed in Table 7, are used in the present study.

The metallicity distributions of the thin and thick disks are also matter of debate. Recent thick disk metallicity determinations quoted mean values from -0.36 (Bell 1996) to -0.70 (Robin et al. 1996, Gilmore et al. 1995), passing by -0.48 (Soubiran et al. 2003). Morrison et al. (1990) brought to the fore low-metallicity stars $(-1.6<[\mathrm{Fe} / \mathrm{H}]<-1.0)$ with disk-like kinematics. Chiba \& Beers (2000) estimate that $30 \%$ of the stars with $-1.6<[\mathrm{Fe} / \mathrm{H}]<-1.0$ belong to the thick disk 


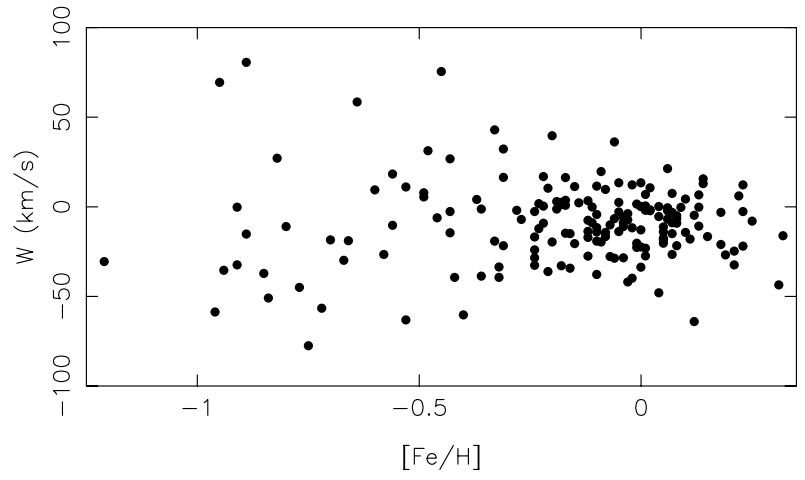

Fig. 6. Distribution of the 174 stars in the plane metallicity $-W$ velocity.

population. Similarly, Bensby et al. (2003) found in their sample stars with super-solar metallicities and thick disk kinematics. It remains unclear whether these extreme populations are separate from the thick disk or their metal-weak and metal-rich tails. Moreover, due to the great overlap of the thin disk and thick disk distributions, it is very difficult to estimate where the transition occurs. Authors involved in the study of metallicity distributions are often concerned with the chemical evolution of the Galaxy and generally do not attempt to separate the thin and thick disks but rather consider the thick disk as an integral part of the disk. It was the case for instance in the work of Edvardsson et al. (1993) on the age - metallicity relation. Haywood (2001) performed a revision of the solar neighbourhood metallicity distribution and concluded that it peaks at $[\mathrm{Fe} / \mathrm{H}]=0$ and that only $4 \%$ of the nearby stars have $[\mathrm{Fe} / \mathrm{H}]<-0.50$. These considerations made us think that the selection of thin disk and thick disk stars on a pure kinematical criterion would be more reliable.

Figure 6 shows the distribution of the sample in the plane metallicity $-W$ velocity. It is clear from this plot that a transition occurs at $[\mathrm{Fe} / \mathrm{H}] \sim-0.30$ : above this value we measure a dispersion of the $W$ velocities of $16 \mathrm{~km} \mathrm{~s}^{-1}$ while below this value the dispersion is $38 \mathrm{~km} \mathrm{~s}^{-1}$. These values are typical of the thin disk and the thick disk respectively (see Table 7). They are related to the different scale heights of the two populations and testify that our sample is indeed a mixture of thin and thick disk stars.

In order to compute the probability of each star to belong to the thin disk or the thick disk on the basis of its spatial velocity, we need to know the kinematical parameters of the two disks and their proportion in our sample. The kinematical parameters are listed in Table 7, but the proportions in our sample are of course different of the real proportion in the solar neibourhood because our selection in the range $-1<[\mathrm{Fe} / \mathrm{H}]<+0.3$ is supposed to have increased the number of thick disk stars comparatively to their relative density in the solar neighbourhood ( $2 \%$ to $15 \%$ ). Moreover the ELODIE library was build from various observing programs which may have biased its content and consequently our sample.

To estimate the proportion of thin and thick disk stars in our sample, we have applied an algorithm of deconvolution of multivariate gaussian distributions, on $(U, V, W)$ velocities. This

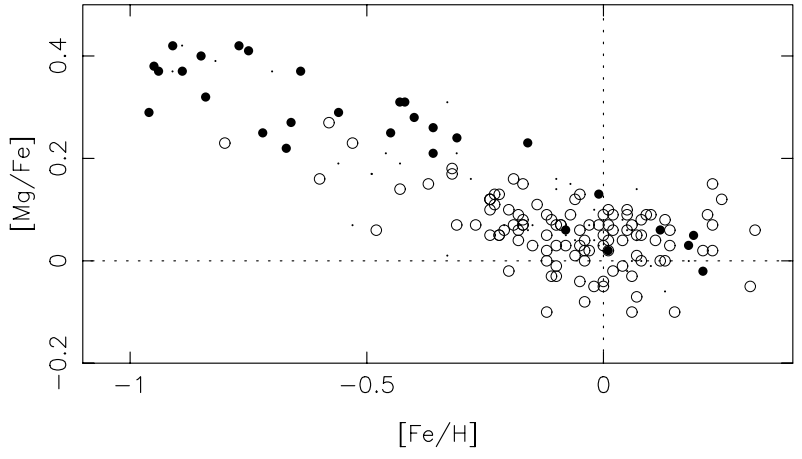

Fig. 7. $[\mathrm{Mg} / \mathrm{Fe}]$ vs. $[\mathrm{Fe} / \mathrm{H}]$ for the whole sample. Black dots indicate thick disk stars, open dots thin disk stars, small dots represent the unclassified stars.

algorithm, SEM (Celeux \& Diebolt 1986), solves iteratively the maximum likelihood equations, with a stochastic step, with no a priori information on the mixture of the gaussian distributions. The results are unambiguous, our sample is consistent with a mixture of 2 Gaussian populations with parameters very similar to those listed in Table 7, but with relative densities of $75 \%$ and $25 \%$ respectively for the thin disk and the thick disk. Accordingly, we have computed the probability of each star, with a measured velocity $(U, V, W)$, to belong to the thin disk $\left(P r_{1}\right)$ and to the thick disk $\left(P r_{2}\right)$ :

$P r_{1}=p_{1} \frac{F_{1}}{F}, P r_{2}=p_{2} \frac{F_{2}}{F}$

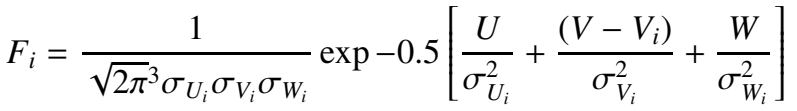

$F=p_{1} F_{1}+p_{2} F_{2}$

where $p_{1}=0.75, p_{2}=0.25$ are the relative densities of the 2 populations in our sample and $V_{i}, \sigma_{U_{i}}, \sigma_{V_{i}}, \sigma_{W_{i}}, i=1,2$ are the kinematical parameters of the thin and thick disks listed in Table 7.

From these probabilities we have selected two subsamples kinematically representative of each population in order to highlight their respective properties. Putting the limit at $\operatorname{Pr}_{i}>80 \%$ ensures a minimal contamination of each subsample by the other population. The thin disk subsample includes 109 stars whereas the thick disk subsample include 30 stars. In the figures, unclassified stars are represented by small dots, thick disk stars by larger black dots, thin disk stars by open dots. The only halo star of the sample, HD 194598 $\left([\mathrm{Fe} / \mathrm{H}]=-1.21, \mathrm{~V}=-276.40 \mathrm{~km} \mathrm{~s}^{-1}\right)$ is not represented in the plots for a better clarity.

Figures 7-9 show the trend of the abundances of $\mathrm{Mg}, \mathrm{Si}$ and $\mathrm{Ni}$ as a function of $[\mathrm{Fe} / \mathrm{H}]$. It can be seen that:

- There are a few thin disk stars with $[\mathrm{Fe} / \mathrm{H}]<-0.30$;

- metal poor stars $([\mathrm{Fe} / \mathrm{H}]<-0.60)$ are all enriched in $\mathrm{Mg}$ and $\mathrm{Si}([\mathrm{Mg} / \mathrm{Fe}]>+0.20,[\mathrm{Si} / \mathrm{Fe}]>+0.15)$;

- on the contrary at solar metallicity, the enrichment of $\mathrm{Mg}$ and $\mathrm{Si}$ does not exceed +0.20 ;

- at a given metallicity thick disk stars have higher $[\mathrm{Mg} / \mathrm{Fe}]$ on average than thin disk stars;

- the dispersion of $[\mathrm{Si} / \mathrm{Fe}]$ is remarkably small for $[\mathrm{Fe} / \mathrm{H}]>-0.30$ but quite high at lower metallicity; 


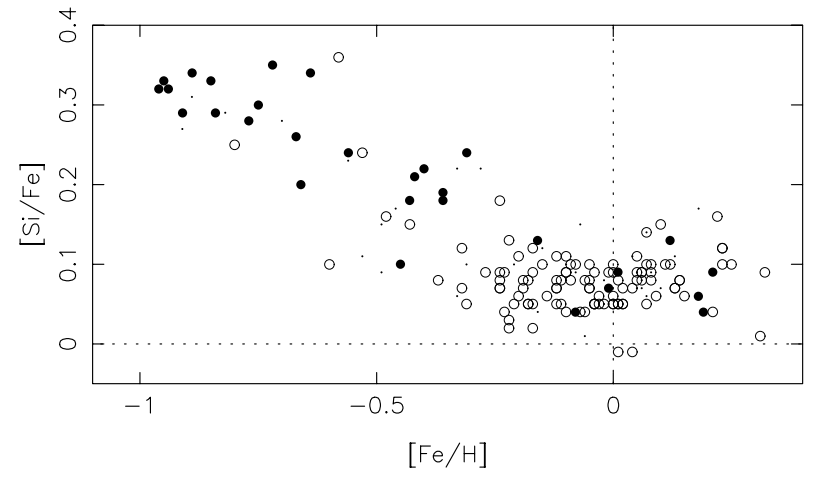

Fig. 8. Same as Fig. 7 for Si.

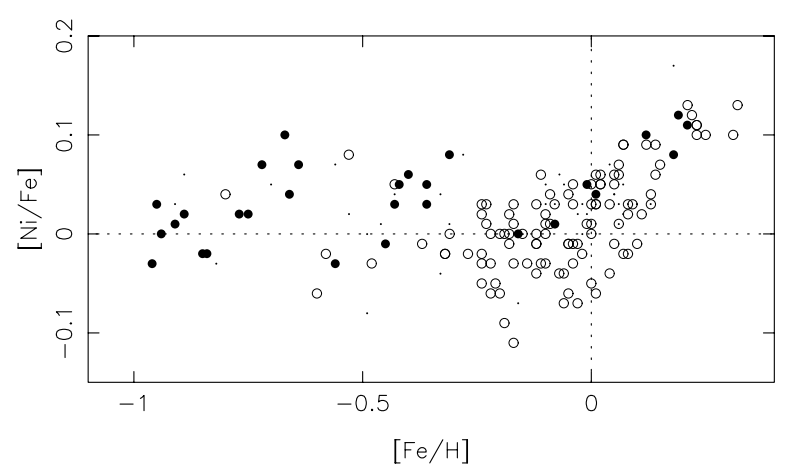

Fig. 9. Same as Fig. 7 for Ni.

- $[\mathrm{Mg} / \mathrm{Fe}]$ and $[\mathrm{Si} / \mathrm{Fe}]$ decline with metallicity from about $+0.40,+0.35$ to $0.0,+0.08$ respectively;

- there are stars with thick disk kinematics at solar metallicities, their abundance trends follow the thin disk;

- a rise and upturn is visible for $[\mathrm{Ni} / \mathrm{Fe}]$ for $[\mathrm{Fe} / \mathrm{H}]>0$.

All these features are in very good agreement with those described in Bensby et al. (2003).

Figure 10 represents the correlation between $\mathrm{Mg}$ and $\mathrm{Si}$ relative to iron. $\mathrm{Mg}$ and $\mathrm{Si}$ are $\alpha$ elements which are supposed to be mainly produced in SNII. It can be seen in this plot that a transition occurs at $[\mathrm{Mg} / \mathrm{Fe}] \simeq+0.2$. Stars with $[\mathrm{Mg} / \mathrm{Fe}]<+0.2$ have a mean abundance of $[\mathrm{Si} / \mathrm{Fe}]=+0.08$ with a very low dispersion of \pm 0.03 , lower than our error estimates. $[\mathrm{Mg} / \mathrm{Fe}]$ is more dispersed $( \pm 0.06)$ around the mean of $[\mathrm{Mg} / \mathrm{Fe}]=+0.05$. For stars with $[\mathrm{Mg} / \mathrm{Fe}]>+0.2$ the distribution is consistent with a linear correlation: $[\mathrm{Si} / \mathrm{Fe}]=$ $0.7[\mathrm{Mg} / \mathrm{Fe}]+0.06(\mathrm{rms}=0.06)$.

\section{Discussion}

\subsection{Stars with thick disk kinematics and thin disk metallicity}

A remarkable feature in the Figs. 7 to 10 is the behavior of stars with thick disk kinematics and thin disk metallicity. They follow exactly the chemical trend of the thin disk. This led us to wonder if these stars were real thick disk stars. According to the $W$ vs. $[\mathrm{Fe} / \mathrm{H}]$ distribution shown in Fig. 6, metal-rich stars are expected to have a smaller scale height than metal poor stars. To have confirmation, we have computed the orbital parameters of

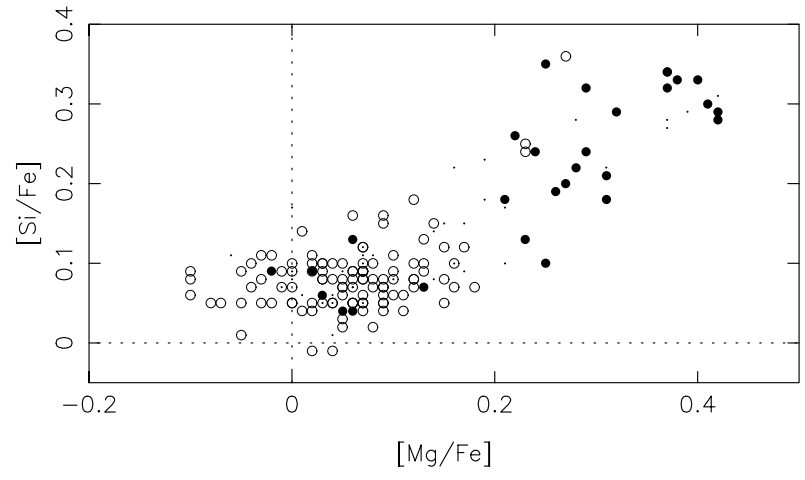

Fig. 10. Correlation between $[\mathrm{Mg} / \mathrm{Fe}]$ and $[\mathrm{Si} / \mathrm{Fe}]$.

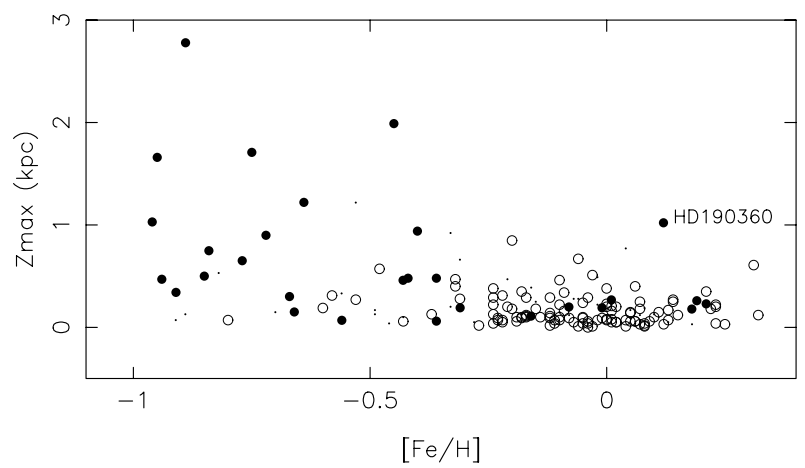

Fig. 11. The maximum distance to the plane of orbits, $Z_{\max }$, versus $[\mathrm{Fe} / \mathrm{H}]$.

our stars, by integrating the equations of motion in the galactic model of Allen \& Santillan (1991) over an age of 8 Gyr. The adopted velocity of the Sun with respect to the LSR is (9, $5,6) \mathrm{km} \mathrm{s}^{-1}$, the solar galactocentric distance $R_{\odot}=8.5 \mathrm{kpc}$ and circular velocity $V_{\mathrm{lsr}}=220 \mathrm{~km} \mathrm{~s}^{-1}$. Table 8 lists the spatial velocities and the parameters of the orbits: apogalactic and perigalactic distances $\left(R_{\min }, R_{\max }\right)$, the maximum distance from the plane $\left(Z_{\max }\right)$ and eccentricity $(e)$. The distribution of $Z_{\max }$ vs. $[\mathrm{Fe} / \mathrm{H}]$ in Fig. 11 confirms the small scale height of stars with thick disk kinematics and solar metallicity, ruling them out of the thick disk. They have been classified into the thick disk because of they have a large eccentricity, but they have a scale height which is typical of the thin disk. Thus the origin of these stars cannot be in the thick disk neither in the local thin disk despite the same chemical behaviour. The origin of these stars has to be found elsewhere. The only ambiguous star, HD 190360 having $[\mathrm{Fe} / \mathrm{H}]=+0.12$ and $Z_{\max }=1 \mathrm{kpc}$, appears as an outlier in the distribution. This star is discussed farther.

Among the 8 stars that verify $\mathrm{Pr}_{2}>80 \%$ and $[\mathrm{Fe} / \mathrm{H}]>-0.30$, HD 030562, HD 139323, HD 139341 are $\mathrm{K}$ dwarfs with very similar velocities and metallicities (Table 8, $+0.18 \leq[\mathrm{Fe} / \mathrm{H}] \leq+0.21)$. HD 030562 has been identified to be part of the HR 1614 moving group by Feltzing \& Holmberg (2000). It is very likely that we have discovered 2 new candidates of this moving group whose age is estimated to be $2 \mathrm{Gyr}$. The origin of this group is however unknown.

HD 010145, HD 135204, HD 152391 have $|U|>$ $80 \mathrm{~km} \mathrm{~s}^{-1}$. An hypothesis is that they have been thrown out from the inner disk by the galactic bar. The over density of 


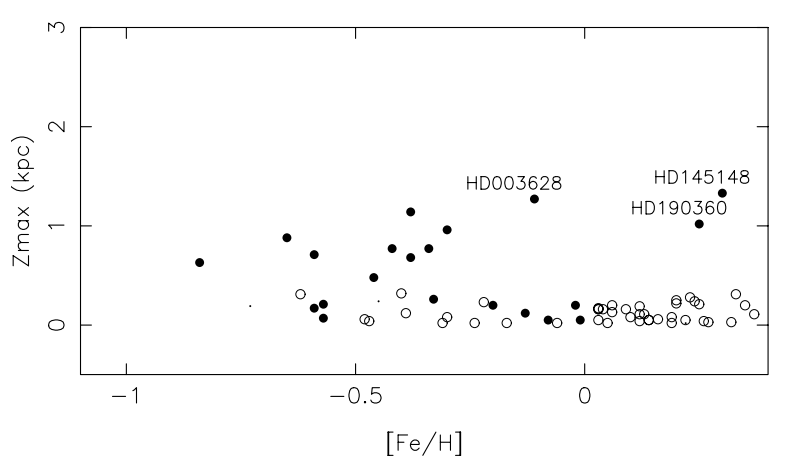

Fig. 12. The maximum distance to the plane of orbits, $Z_{\max }$, versus $[\mathrm{Fe} / \mathrm{H}]$ for Bensby et al.'s sample. The classification thin disk/thick disk is the same as ours $\left(P r_{i}>80 \%\right)$.

metal-rich stars with excentric orbits confined near the plane have have been interpreted as the signature of the galactic bar in the solar neighbourhood by Raboud et al. (1998) for instance.

Bensby et al. (2003) have also found several stars with thick disk kinematics and thin disk metallicity on the basis of a similar probability calculation than ours, but using the ratio of $P r_{2}$ to $P r_{1}$. If in our case these stars have a clearly a flatter distribution than the thick disk, it was not mentioned in Bensby et al.'s study. As it is important to demonstrate if they belong or not to the thick disk we have calculated the orbits of their stars and classified them with the same criterion as ours $\left(\operatorname{Pr}_{i}>80 \%\right)$. The distribution of $Z_{\max }$ versus $[\mathrm{Fe} / \mathrm{H}]$ is shown on Fig. 12. Globally their distribution is similar to ours despite their sample is much smaller. There is a sudden rise in $Z_{\max }$ at $[\mathrm{Fe} / \mathrm{H}]<-0.3$. They have also 3 outliers with solar metallicities and $Z_{\max } \sim 1 \mathrm{kpc}$, HD 190360 being in common with us. The five other stars with thick disk kinematics and $[\mathrm{Fe} / \mathrm{H}]>-0.30$ have a low scale height typical of the thin disk in agreement with our findings. The question wether HD 190360, HD 003648 and HD 145148 are really part of the thick disk is an open question because they appear as outliers in the distribution of kinematics vs. metallicity. It is only with complete samples with no kinematical bias that the relation of such stars with the thick disk will be clarified.

\subsection{The thin disk}

Several thin disk stars are found at low metallicity, down to $[\mathrm{Fe} / \mathrm{H}]=-0.80$. Several previous studies have also established the existence of such stars, however the exact contribution of metal-poor stars to the thin disk is difficult to evaluate from uncomplete samples. Reddy et al. (2002) have found in their samples a significant number of stars with $[\mathrm{Fe} / \mathrm{H}]<-0.40$ that they identify as belonging to the thin disk. They have analysed 181 FG dwarfs, a sample size similar to ours. Their sample spans the metallicity interval -0.70 to +0.15 , with a peak at $[\mathrm{Fe} / \mathrm{H}]=-0.40$. Only 3 stars were identified as belonging to the thick disk, based on the criterion $[\mathrm{Mg} / \mathrm{Fe}]>+0.20$, all the other stars being identified as thin disk stars. The peak at $[\mathrm{Fe} / \mathrm{H}]=-0.40$ shows clearly that they have favoured in some way the metal-poor tail of the thin disk. Unfortunatly their selection criteria are not described. On the contrary, the way Chen et al. (2000) have favoured metal-poor thin disk stars in their sample is very clear: they have selected equal number of stars in every metallicity interval of $0.1 \mathrm{dex}$ in the range $-1.0<[\mathrm{Fe} / \mathrm{H}]<+0.3$. According to the low density of the thick disk in the solar neighbourhood, thin disk stars are still dominating at moderatly low metallicity. Haywood (2001) has very carefully taken into account the selection biases of his sample to study the metallicity distribution of the local disk but his metallicities are photometric and no special attention was paid to a kinematical distinction between the thin disk and the thick disk. Nevertheless he found, contrary to Wyse \& Gilmore (1995), a low contribution of the thin disk at $[\mathrm{Fe} / \mathrm{H}]<-0.40$.

Our observations suggest that the distribution of $[\alpha / \mathrm{Fe}]$ in the thin disk is very narrow, specially for $\mathrm{Si}$, at $[\mathrm{Fe} / \mathrm{H}]>-0.30$. On this point we are in perfect agreement with Reddy et al. (2002) and Bensby et al. (2003). For the metal-rich part, we obtain as mean values and dispersions: $[\mathrm{Mg} / \mathrm{Fe}]=+0.05$, $\sigma_{[\mathrm{Mg} / \mathrm{Fe}]}=0.07,[\mathrm{Si} / \mathrm{Fe}]=+0.07, \sigma_{[\mathrm{Si} / \mathrm{Fe}]}=0.03$. Such a narrow chemical distribution implies that the stars formed from an homogeneous gas. At lower metallicity, the $\mathrm{Mg}$ enhancement is higher. However at a given metallicity the thin disk shows a lower $\mathrm{Mg}$ enhancement than the thick disk. The behaviour of $\mathrm{Si}$ is quite surprising, showing high dispersion, whereas Bensby et al. (2003) find a similar behaviour than Mg. We notice also that the Sun would be slightly Si-poor as compared to the thin disk. However this offset stays within the error bars and might be related to the adopted zero point of our abundance scales. Many previous studies (Edvardsson et al. 1993; Chen et al. 2000; Reddy et al. 2002; Bensby et al. 2003) exhibit also a positive offset in $[\mathrm{Si} / \mathrm{Fe}]$ of the thin disk.

A rise of $[\mathrm{Ni} / \mathrm{Fe}]$ from $\sim-0.1$ at $[\mathrm{Fe} / \mathrm{H}]=-0.20$ to $\sim+0.1$ at $[\mathrm{Fe} / \mathrm{H}]=+0.20$ is visible in Fig. 9 as well as an upturn for the most metal rich star. A similar feature is also observed in Bensby et al. (2003).

\subsection{The thick disk}

Having eliminated from the thick disk the 8 stars having high metallicity and a flat distribution (Sect. 7.1), we see from Fig. 7 that $[\mathrm{Mg} / \mathrm{Fe}]$ decreases from the halo value $(+0.40)$ at $[\mathrm{Fe} / \mathrm{H}]=-1.00$ to +0.20 at $[\mathrm{Fe} / \mathrm{H}]=-0.30$. Si has the same behaviour, decreasing from +0.35 to +0.17 , with one deviating star HD 110897 having a lower enrichment in Si. These findings are nicely consistent with the trends found by Bensby et al. (2003) that they interpreted as the signature of the chemical enrichment by SNIa. Feltzing et al. (2003) claimed the existence of a "knee" in the $[\alpha / \mathrm{Fe}]$ abundance trends tracing the beginning of the contribution of the SNIa. This "knee" may also exist in our data, especially in $[\mathrm{Mg} / \mathrm{Fe}]$ but the dispersion is too high to conclude. Definitively a larger sample of thick disk stars is necessary to clarify this point. Contrary to Bensby et al. (2003) and Feltzing et al. (2003), our data suggest that the metallicity distribution of the thick disk does not continue at solar metallicity. The trend of declining ehancement, which was also mentioned in Prochaska et al. (2000), has to be compared to the results of Fuhrmann (1998) and Gratton et al. (2000) who found in the thick disk a constant overabundance of 


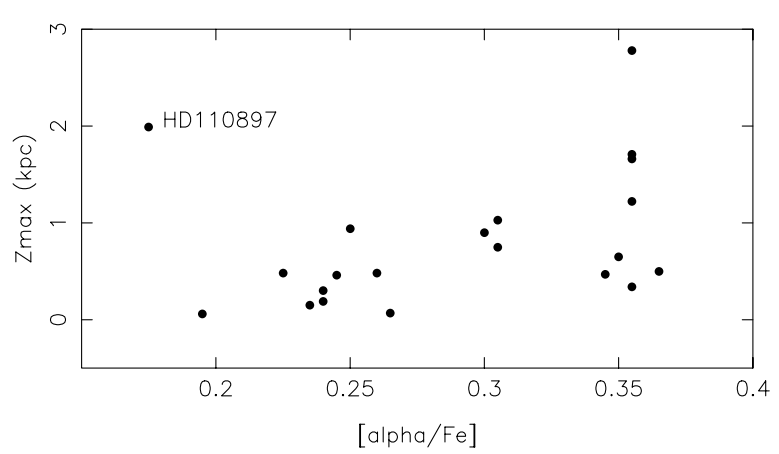

Fig. 13. A possible vertical gradient in $[\alpha / \mathrm{Fe}]$ in the thick disk?

$\alpha$-elements with respect to iron, with a value similar to that of the halo. In Fuhrmann (1998), only thin disk stars exhibit a decrease of $[\mathrm{Mg} / \mathrm{Fe}]$ with increasing $[\mathrm{Fe} / \mathrm{H}]$. We believe that we have been able to detect this trend, as Feltzing et al. (2003) and Bensby et al. (2003), because we have used a more precise criterion to isolate a sample a pure thick disk stars. We agree with most of the previous works that a discontinuity exists between the chemical distributions of the thin and thick disks. Our data suggest that the star formation in the thick disk stopped when the gas had the composition $[\mathrm{Fe} / \mathrm{H}]=-0.3,[\mathrm{Mg} / \mathrm{Fe}]=+0.20$, $[\mathrm{Si} / \mathrm{Fe}]=+0.17$.

The distribution of $Z_{\max }$ vs. [Fe/H] (Fig. 11) does not exhibit a clear vertical gradient in the thick disk metallicity. We have searched for a vertical gradient in the $\alpha$-element abundances. Figure 13 represents $Z_{\max }$ vs. $[\alpha / \mathrm{Fe}]=0.5([\mathrm{Mg} / \mathrm{Fe}]+$ $[\mathrm{Si} / \mathrm{Fe}])$ for the thick disk $\left(\mathrm{Pr}_{2}>0.80\right.$ and $\left.[\mathrm{Fe} / \mathrm{H}]<-0.3\right)$. A gradient may exist, but should be confirmed with a larger sample. The existence of a vertical gradient would imply a significant timescale for the formation of the thick disk. This implication is also true if the decline of $\alpha$ enhancement is interpreted by the onset of SNIa.

\section{Summary and perspectives}

We have investigated the chemical behaviour of the thin disk and the thick disk with a large sample of FGK dwarfs spanning the metallicity interval $[-1.00 ;+0.30]$. We have used an homogeneous set of high resolution, high $S / N$ echelle spectra to derive abundances of $\mathrm{Fe}, \mathrm{Mg}, \mathrm{Si}$ and Ni with detailed NLTE calculations for Mg. All sources of errors have been analysed and we have compared our abundances with several studies taken in the literature, showing a good agreement. Orbits and space velocities were computed and used to estimate the probability of each star to belong to the thin disk and the thick disk, taking into account the selection bias that affects our local sample where the thick disk is overrepresented as compared to the solar neighbourhood. Our main findings concern the transition in kinematics and abundance trends which occurs at $[\mathrm{Fe} / \mathrm{H}]=-0.30$, the decline of $\alpha$ enhancement with increasing $[\mathrm{Fe} / \mathrm{H}]$ in the thick disk, and the narrow distribution of $\mathrm{Mg}$ and $\mathrm{Si}$ in the thin disk. We have also observed several stars which cannot belong to the thin disk because of their large eccentricity, neither to the thick disk because of their low scale height. This population, perhaps coming from the inner disk due to a dynamical effect, has to be taken into account when studying the metallicity distributions of the stellar populations in the solar neighbourhood. Among these stars we have discovered two new candidates of the HR 1614 moving group.

Larger samples are necessary to confirm our findings on the thin disk and the thick disk. Unfortunatly thick disk stars are quite rare in the solar neighbourhood and the construction of a significant sample of local thick disk stars implies either a huge complete sample of disk stars, among which $2 \%$ to $15 \%$ of thick disk stars are expected, or the selection of such stars by kinematical or chemical criteria which bias the conclusions which can be drawn. A lot of observing material is available. In the public ELODIE library, there are hundreds of high quality spectra of FGK stars that have not been analysed yet. Most of these stars have accurate parallaxes and proper motions from Hipparcos and their radial velocities are also available. It is thus possible to compute their probability to belong to a given kinematical population. More difficult but necessary is to evaluate the biases which render the metal-poor and high velocity stars more represented in the library than in the solar neighbourhood, reflecting the interest of observers in this kind of stars. Another solution to avoid these biases is to observe the thick disk in situ that is at distances and galactic latitudes where it begins to dominate the thin disk in density. We are continuing such an observing program (Soubiran et al. 2003).

Detailed analysis of spectroscopic data is a tedious work that takes much time to measure equivalent widths, to fit profiles, to compute models etc. It is now absolutly necessary to develop automatic methods to take advantage of the huge amount of high quality data which is provided by the new generation of spectrographs. We have undertaken such work to continue our investigation of the correlation between elemental abundances and kinematics among galactic disk stars from larger, more distant and complete samples.

Acknowledgements. T.M. wants to thank the Observatoire de Bordeaux for kind hospitality. Our special thanks to the referee Dr. M. Asplund for fruitful comments and suggestions. This research has made use of the SIMBAD and VIZIER databases, operated at CDS (Strasbourg, France) and ESA products (Hipparcos catalogue).

\section{References}

Allen, C. W. 1973, Astrophysical Quantities (London: Athlone Press) Allen, C., \& Santillan, A. 1991, Rev. Mex. Astron. Astrofis., 22, 255 Allende Prieto, C., García López, R. J., Lambert, D. L., \& Gustafsson, B. 1999, ApJ, 527, 879

Alonso, A., Arribas, S., \& Martínez-Roger, C. 1996, A\&AS, 117, 227 Asplund, M. 2000, A\&A, 359, 755

Asplund, M., Nordlund, A., Trampedach, R., \& Stein, R. F. 2000, A\&A, 359, 743

Asplund, M. 2003, in Highlights of Astronomy, ed. P. E. Nissen, \& M. Pettini, 13

Baranne, A., Queloz, D., Mayor, M., et al. 1996, A\&AS, 119, 373

Barklem, P. S., Piskunov, N., \& O'Mara, B. J. 2000, A\&AS, 142, 467 Bell, D. C. 1996, Ph.D. Thesis, Univ. of Illinois

Bensby, T., Feltzing, S., \& Lundstrom, I. 2003, A\&A, 410, 527

Biemont, E., \& Brault, J. W. 1986, Phys. Scr., 34, 751

Blackwell, D. E., \& Lynas-Gray, A. E. 1998, A\&A, 129, 505

Blackwell, D. E., Lynas-Gray, A., \& Smith, G. 1995, A\&A, 296, 217

Burbidge, E. M., Burbidge, G. R., Fower, W. A., \& Hoyle, F. 1957, Rev. Mod. Phys., 29, 547 
Carlsson, M. 1986, Uppsala Obs. Rep., 33

Celeux, G., \& Diebolt, J. 1986, Rev. Stat. Appl., 35, 36

Chen, Y. Q., Nissen, P. E., Zhao, G., et al. 2000, A\&AS, 141, 491

Chiba, M., \& Beers, T. C. 2000, AJ, 119, 2843

di Benedetto, G. P. 1998, A\&A, 339, 858

ESA 1997, The HIPPARCOS and TYCHO catalogues (Noordwijk, Netherlands: ESA Publications Division)

Drawin, H. W. 1968, Z. Physik, 211, 404

Edvardsson, B., Andersen, J., Gustafsson, B., et al. 1993, A\&A, 275, 101

Feltzing, S., Bensby, T., \& Lundstrom, I. 2003, A\&A, 397, L1

Feltzing, S., \& Holmberg, J. 2000, A\&A, 357, 153

Fuhrmann, K., Axer, M., \& Gehren, T. 1995, A\&A, 301, 492

Fuhrmann, K. 1998, A\&A, 338, 161

Fulbright, J. P. 2000, AJ, 120, 1841

Galazutdinov, G. A. 1992, Preprint SAO RAS, 92, 27

Gehren, T., Butler, K., Mashonkina, L., Reetz, J., \& Shi, J. 2001a, A\&A, 366, 981

Gehren, T., Korn, A. J., \& Shi, J. 2001b, A\&A, 380, 645

Gilmore, G., Wyse, R. F. G., \& Jones, J. B. 1995, AJ, 109, 1095

Gratton, R. G., \& Sneden, C. 1987, A\&A, 178, 179

Gratton, R. G., Carretta, E., \& Castelli, F. 1996, A\&A, 314, 191

Gratton, R. G., Carretta, E., Eriksson, K., \& Gustafsson, B. 1999, A\&A, 350, 955

Gratton, R. G., Carretta, E., Matteucci, F., \& Sneden, C. 2000, A\&A, 358,671

Gratton, R., Carretta, E., Claudi, R., Lucatllo, S., \& Barbieri, M. 2003, A\&A, 404, 187

Gray, D. 1994, PASP, 106, 1248

Grevesse, N., \& Sauval, A. J. 1998, Space Sci. Rev., 85, 161

Haywood, M. 2001, MNRAS, 325, 1365

Heiter, U., \& Luck, R. E. 2003, AJ, 126, 2015

Hirata, R., \& Horaguchi, T. 1994, Atomic spectral line list, CDS VI/69

Idiart, T. P., \& Thévenin, F. 2000, ApJ, 541, 207

Katz, D., Soubiran, C., Cayrel, R., Adda, M., \& Cautain, R. 1998, A\&A, 338, 151

Korotin, S. A., Andrievsky, S. M., \& Luck, R. E. 1999, A\&A, 351, 168

Korotin, S. A., Andrievsky, S. M., \& Kostynchuk, L. Yu. 1999, Ap\&SS, 260, 531

Kovtyukh, V. V., \& Andrievsky, S. M. 1999, A\&A, 351, 597

Kovtyukh, V. V., Soubiran, C., Belik, S. I., \& Gorlova, N. I. 2003, A\&A, 411, 559

Kurucz, R. L. 1993, CD ROM No. 13

Kurucz, R. L., Furenlid, I., Brault, J., \& Testerman, L. 1984, Solar Flux Atlas from 296 to $1300 \mathrm{~nm}$
Kurucz, R. L. 1992, The Stellar Populations of Galaxies, ed. B. Barbuy, \& A. Renzini, IAU Symp., 149, 225

Magain, P. 1989, A\&A, 209, 211

Maltby, P., Avrett, E. H., Carlsson, M., et al. 1986, ApJ, 306, 284

Martin, W. C., \& Zalubas, R. 1980, J. Phys. Chem. Ref. Data, 9, 1

Mashonkina, L., \& Gehren, T. 2000, A\&A, 364, 249

Mashonkina, L., \& Gehren, T. 2001, A\&A, 376, 232

Mashonkina, L., Gehren, T., Travaglio, C., \& Borkova, T. 2003, A\&A, 397,275

Mishenina, T. V., \& Kovtyukh, V. V. 2001, A\&A, 370, 951

Morrison, H. L., Flynn, C., \& Freeman, K. C. 1990, AJ, 100, 1191

Nissen, P. E., Gustafsson, B., Edvardsson, B., \& Gilmore, G. 1994, A\&A, 285, 440

Norris, J. E. 1999, Ap\&SS, 265, 213

Prochaska, J. X., Naumov, S. O., Carney, B. W., McWilliam, A., \& Wolfe, A. M. 2000, AJ, 120, 2513

Prugniel, P., \& Soubiran, C. 2001, A\&A, 369, 1048

Raboud, D., Grenon, M., Martinet, L., Fux, R., \& Udry, S. 1998, A\&A, 335, L61

Reddy, B. E., Tomkin, J., Lambert, D. L., \& Prieto, C. A. 2002, MNRAS, 340, 304

Reid, N., \& Majewski, S. R. 1993, ApJ, 409, 635

Reylé, C., \& Robin, A. C. 2001, A\&A, 373, 886

Robin, A. C., Haywood, M., Crézé, M., Ojha, D. K., \& Bienaymé, O. 1996, A\&A, 305, 125

Shchukina, N., \& Bueno, J. Tr. 2001, ApJ, 550, 970

Shimanskaya, N. N., Mashonkina, L. I., \& Sakhibullin, N. A. 2000, ARep, 44, 530

Seaton, M. J. 1962, Proc. Phys. Soc., 79, 1105

Soubiran, C., Katz, D., \& Cayrel, R. 1998, A\&AS, 133, 221

Soubiran, C., Bienaymé, O., \& Siebert, A. 2003, A\&A, 398, 141

Steenbock, W., \& Holweger, H. 1984, A\&A, 130, 319

Thévenin, F., \& Idiart, T. P. 1999, ApJ, 521, 753

Timmes, F. X., Woosley, S. E., \& Weaver, T. A. 1995, ApJS, 98, 617

Van Regemorter, H. 1962, ApJ, 136, 906

Unsöld, A. 1955, Physik der Sternatmosphären, 2nd ed. (Berlin: Springer-Verlag)

Voronov, G. S. 1997, ADNDT, 65, 1

Wallerstein, G. 1961, ApJS, 6, 407

Wedemeyer, S. 2001, A\&A, 373, 998

Wyse, R. F. G., \& Gilmore, G. 1995, AJ, 110, 2771

Yan, Y., Taylor, K. T., \& Seaton, M. J. 1987, J. Phys. B: At. Molec. Phys., 20, 6409

Zboril, M., \& Byrne, P. B. 1998, MNRAS, 299, 753

Zhao, G., \& Gehren, T. 2000, A\&A, 362, 1077 